\title{
A Framework for the Automation of LTE Physical Layer Tests
}

\author{
Felipe A. P. de Figueiredo • Fabiano \\ Mathilde • Dick Carrillo • Ingrid \\ Moerman
}

Received: date / Accepted: date

\begin{abstract}
The Long Term Evolution (LTE) network architecture comprises, among other elements, the Base Station (eNodeB), which provides and controls the air interface. ENodeB protocol stack is composed of different layers, each one with specific purpose. In terms of air interface performance, the most critical layers of an eNodeB are the physical layer (PHY) and the medium access control Layer (MAC). Both must operate on precise timing basis, corresponding to a frame duration of $1 \mathrm{~ms}$. Because of this requirement, PHY layer developers need an effective test architecture, capable to follow time response policies. This article proposes an automated test framework for eNodeB's physical layer development, comprising procedures for checking data integrity, stability and performance. This framework is based on a simplified LTE MAC layer, which operates as a software element that communicates directly with the physical layer and performs mapping procedures between logical and physical channels, reception and transmission of physical layer data, user data scheduling and data exchange with mobile terminals. All above mentioned procedures are performed with no further dependency on other LTE network elements, thus providing a stand-alone test framework.
\end{abstract}

Keywords LTE, · Physical Layer · Test Framework · MAC

\author{
Felipe A. P. de Figueiredo · Ingrid Moerman \\ Ghent University - imec, IDLab, Department of Information Technology, Ghent, Belgium. \\ Fabiano Mathilde \\ Eldorado Institute, Campinas, Brazil \\ Dick Carrillo \\ CPqD - Research and Development Center on Telecommunications, Campinas, Brazil.
}

$\bowtie$ E-mail: felipe.pereira@ugent.be 


\section{Introduction}

The LTE corresponds to one of the most important current mobile communication technologies, and has been standardized by the Third Generation Partnership Project (3GPP) $[1,2]$. LTE was developed to meet the growing demands of data transmission at high speed, also supplying voice transmission. The main advantages of LTE are based on new specifications for radio access network (RAN), achieving high data rates, low latency transmission and high spectral efficiency. Such benefits are only possible due to the architecture defined for the Radio Access Network (RAN), comprising base stations (eNodeB) and user equipment (UE) [1-3].

The eNodeB's protocol architecture comprises several layers [3]. Each layer has a different purpose, according to its operating protocol, and serves different timing requirements [4-8]. Due to advanced communication technologies employed in LTE, such as Orthogonal Frequency Division Multiplexing (OFDM) and Multiple Input Multiple Output (MIMO), some layers become critical for system performance, such as the Physical Layer (PHY), which is often developed as a hardware-level element [9-11]. The second critical layer of eNodeB stack is the Medium Access Control Layer (MAC), which is developed as a high-performance and real-time software element due to its direct communication with physical layer (PHY) [7]. Because of interoperability nature of LTE architecture, PHY and MAC layers are developed by different vendors, generating an integration effort. Based on this fact, this article focuses on a test framework that consists of a simplified MAC layer (MAC) targeting PHY layer conformance, performance and integration tests [11]. Such architecture is designed to operate independently by upper layers, as a stand-alone solution. In order to achieve it, a simplified and light-weight MAC layer (MAC LITE) has been designed to emulate a real MAC layer, covering throughput performance, stability, data integrity and conformance test cases [11]. It has been noticed that MAC LITE is extremely useful for PHY development.

The paper is organized as follows. Section 2 presents the motivation for creating the test framework for physical layer development. Section 3 provides a detailed explanation of eNodeB protocol stack and the architecture proposed for testing, while Section 4 presents the main use cases. Section 5 presents the conclusions and references.

\section{Motivation}

In September 2012, 3GPP started a Working Item to standardize the frequency bands $450-470 \mathrm{MHz}$ [12]. This initiative aimed at taking advantage of $\mathrm{RF}$ propagation characteristics in lower frequency bands, based on a new LTE and LTE-Advanced profile $[12,13]$. This profile is useful in scenarios with large coverage requirements in sparsely populated areas. The $\mathrm{CPqD}$ Foundation supported 3GPP in this task, by determining new channel schemes, solutions for coexistence and radio transceiver parameters suitable for LTE 
and LTE-Advanced operation in the $450 \mathrm{MHz}$ band. The standardization process finished in September 2013, and band 450-470 MHz has been designated as the new 3 GPP Band 31.

In parallel to $3 \mathrm{GPP}$ activities, the Communications Ministry has been working on a way to introduce new broadband Internet services in Brazil [14, 15]. In this sense, a paradigm shift occurred in May 2010, when the National Broadband Plan (PNBL) recommended the use of the frequency band 225-470 $\mathrm{MHz}$ [13]. One of PNBL guidelines was to leverage broadband Internet penetration in Brazilian territory, specifically in rural areas, where about 30 million Brazilians still lack access to this type of service. Also in 2010, the National Telecommunications Agency (ANATEL) has allocated the frequency bands 451-458 MHz and 461-468 MHz to serve as downlink and uplink, respectively, for fixed and mobile radio services operating on Division Duplex mode [13].

Furthermore, ANATEL auctioned the Band 31 licenses in June 2012. As a result, Band 31 is now available to all the major telecommunications operators within in the Brazilian market. It is worth mentioning that the winning operators must meet the coverage and capacity targets defined by ANATEL [13], while the adoption of specific technology associated with these licenses is open. It is believed that the operators holding licenses will most likely adopt LTE technology to take advantage of superior RF features of Band 31 for providing broadband access [12-15].

LTE implementation in Band 31, besides providing wider coverage area per sector, imposes many technical challenges, mostly related to RF characteristics. In order to ensure Band 31 benefits are achieved, eNodeBs require specific technology development, especially related to RF components and PHY features. In this context, a stand-alone framework is proposed, for PHY conformance, stability, data integrity and performance testing. It is composed of a simplified MAC layer (MAC LITE) and main procedures for automated testing. The next section will provide more details of LTE layers and the proposed testing framework.

\section{Fundamentals of LTE Layers}

This section is composed of three distinct parts. The first part discusses the details of eNodeB architecture, showing all its main features [16-19]. The second part discusses in detail the main features of the proposed testing framework, while the third part describes its use cases.

\section{1 eNodeB architecture}

In LTE and LTE-Advanced systems, the eNodeB protocol stack is composed of layers and sub-layers as illustrated in Figure 1. Each layer performs specific functionalities [1], as described below: 


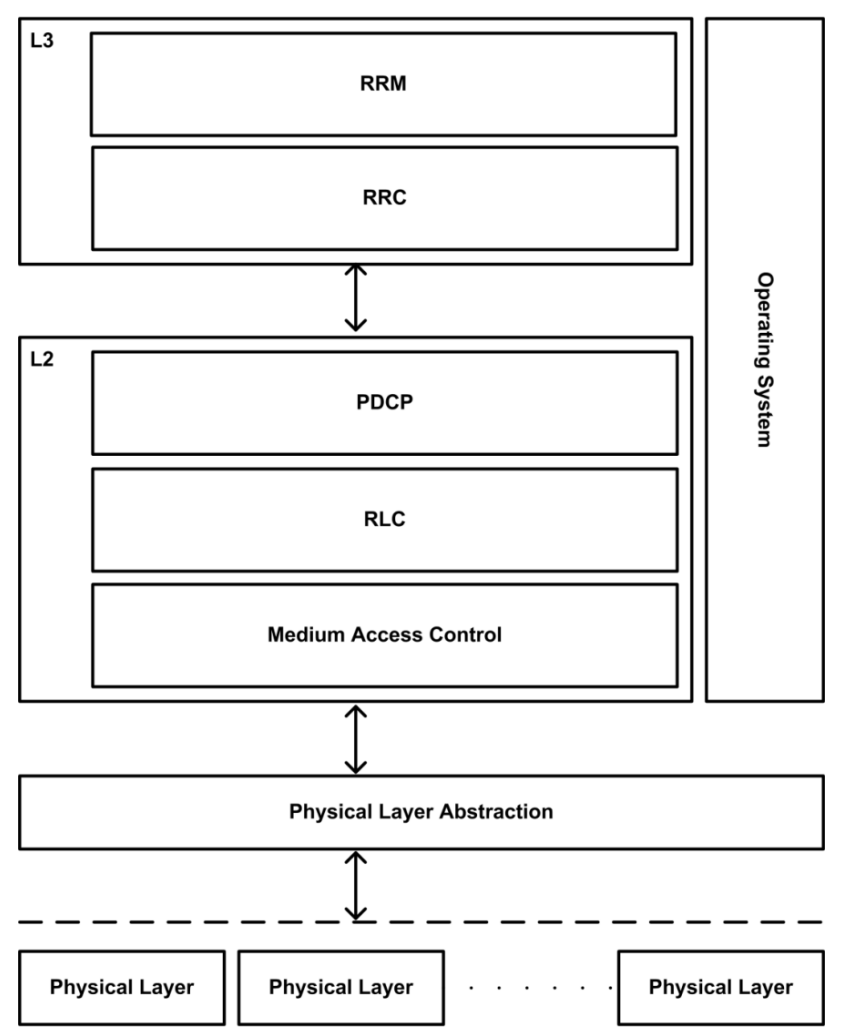

Fig. 1 eNodeB described in 3GPP Standard.

1. Radio Resource Management (RRM): this sub-layer of Layer 3 is responsible for management and efficient usage of radio frequency resources [20]. The RRM supports mechanisms and algorithms for controlling air interface resource, such as power transmission, resource allocation for users, data rates, modulation schemes and error control. All these strategies aim at better usage of limited radio frequency spectrum;

2. Radio Resource Control (RRC): this sub-layer of Layer 3 is responsible for establishment, maintenance and control of connections between user equipment and base stations [8];

3. Packet Data Convergence Control (PDCP): this sub-layer of Layer 2 is responsible for compression and decompression of user data encapsulated by IP protocol, as well as checking data integrity or insert redundancies $[5]$;

4. Radio Link Control (RLC): this sub-layer of Layer 2 is responsible for data packets transference to PDCP layer. The packets containing user data comes from PDCP layers and are encapsulated in RLC packets. Depending on channel conditions, system configuration and packet length, RLC splits original packet into multiple packets, transmitting them according to the system transmission capacity. RLC is also responsible for reordering, con- 
solidation and removing duplicate data packets, which were originated by access layer [6];

5. Medium Access Layer (MAC): this sub-layer of Layer 2 performs the mapping between transport and physical channels. It is also responsible for resource block scheduling and error correction by selective repetition system, also named HARQ (Hybrid ARQ) [7]. The main transport channels are [21]:

(a) Broadcast Channel (BCH): downlink signaling channel used for transmitting broadcast messages, and sending information system to all handsets into eNodeB coverage region;

(b) Downlink Shared Channel (DL-SCH): downlink signaling channel used for transmitting data information to mobile terminals;

(c) Uplink Shared Channel (UL-SCH): uplink data channel, used to transmit data information from mobile terminals to eNodeB;

(d) Random Access Channel (RACH): uplink signaling channel used for transmitting messages related to random access channel procedures between mobile terminals and eNodeBs.

6. Physical Layer (PHY): this layer performs wireless communication with LTE mobile devices, as well as the interface with upper layers [4,10,22]. It supports different transmission characteristics, according to data transmission type. The transmission characteristics are defined by so-called physical channels, which receive data from transport channels [10]. Due to the great complexity and necessity of low-latency communication of physical channels, such layer is usually developed using hardware elements such as FPGA, DSP or ASIC. Each LTE physical channel has a specific purpose, as per the following description $[10,17]$ :

(a) Physical Broadcast Channel (PBCH): Control channel responsible of transporting broadcast messages. It transmits system information to all mobile terminals. It is part of downlink channel structure;

(b) Physical Control Format Indicator Channel (PCFICH): Control channel responsible to transmit mobile terminals information related to format of control channel transmission $(\mathrm{PDCCH})$. It is part of downlink channel structure;

(c) Physical Downlink Control Channel (PDCCH): Control channel responsible of transmitting resource block allocation, power control and paging. It is part of downlink channel structure;

(d) Physical Hybrid ARQ Indicator Channel (PHICH): Control channel responsible to transmit HARQ information to mobile terminals. It is part of downlink channel structure;

(e) Physical Downlink Shared Channel (PDSCH): this channel is responsible to transmit data information from eNodeB to mobile. This is the physical channel with higher throughput. It is part of downlink channel structure; 
(f) Physical Uplink Control Channel (PUCCH): Control channel responsible to transmit signal information related to scheduling request. It is part of uplink channel structure;

(g) Physical Uplink Shared Channel (PUSCH): Channel responsible to transmit data information from mobile terminals to eNodeBs. It is part of uplink channel structure;

(h) Physical Random Access Channel (PRACH): Channel responsible of sending random access preamble that mobile terminal transmits to eNodeB in unsynchronized mode. Such preamble is used so that the UE can synchronize timing with the eNodeB. It is part of uplink channel structure.

All layers discussed above are necessary for complete operation of an eNodeB. The upper layers of the LTE network have not been mentioned since they are not part of this article's scope. The layers above the physical layer (RRM, RRC, PDCP, RLC and access layer) are often developed as real time embedded software elements, because of requirements such us flexibility, scalability, upgradeability, among others. In case of LTE, physical layers often use well-structured interface for communication with the upper layers, such as the well-known Femto API [23] (a direct reference to small and medium-sized stations, also known as Femto Cells). Such interface is based on standardized (PHY) and access layer (MAC) messages. The framework described in this article uses this protocol as a reference for communication and operation of physical layer in testing phase. In the next section it is explained in detail the architecture features for testing proposed by the article.

\subsection{Simplified MAC Layer Architecture}

As mentioned in the introductory section, the proposed architecture has been designed as a stand-alone framework for PHY testing, with no dependency on upper layers described in the previous section. In order to make this possible, the creation of a centralizing element was necessary to carry out the main test procedures based on the user-defined settings. Such software element containing procedures for master boot, injection, recovery of configuration data parameters. These actions are based on procedures described by the Femto Forum API interface [23], since it also includes the main operating and starting procedures of commercial physical layers. For the full operation of such element, the architecture relies on a few layers or intermediate components. Such elements are described in Figure 2.

The whole framework has been designed to run on a computer with a generic operating system. Each component shown in Figure 2 is described below from top to bottom.

1. User Data: in order to test the LTE physical layer, reference data is sent and compared with the received data, after the processing performed by corresponding layer. Therefore, it is possible to check the error rate and 


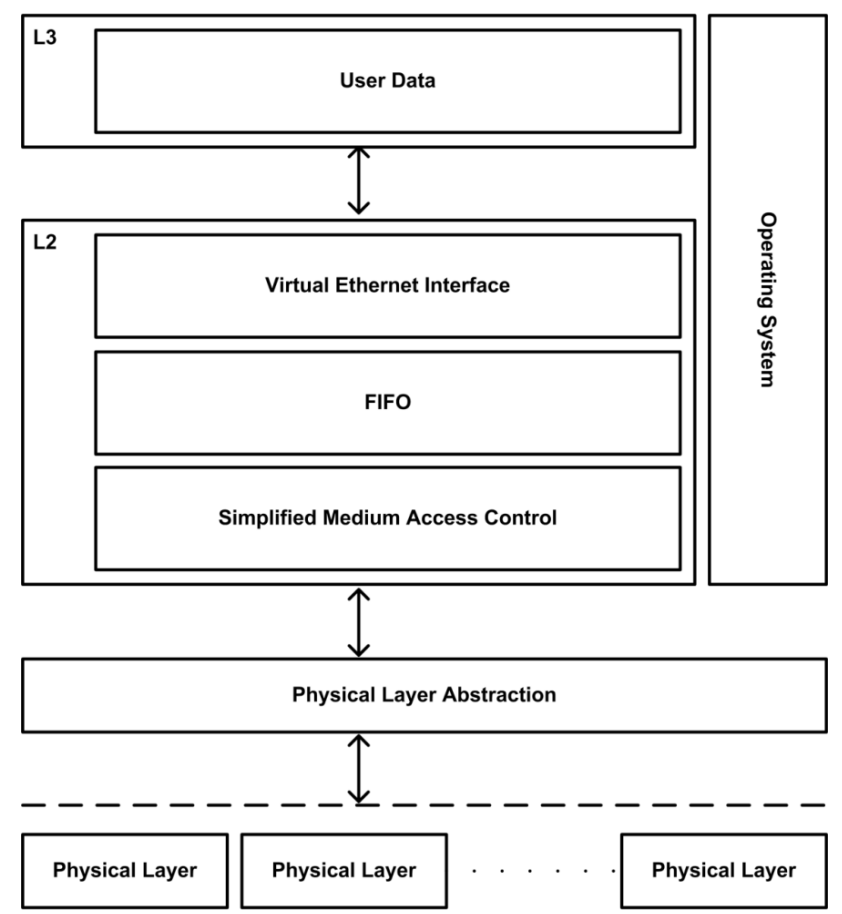

Fig. 2 Proposed Framework Architecture.

the system throughput as a whole. The framework comprises an abstraction at the operating system level, where data is sent and received through a virtual network interface, thus enabling data injection from any application that supports communication via network, facilitating the testing procedure;

2. Virtual Ethernet Interface: the purpose of this interface is to collect reference data and injecting it into the system. All modern operating systems support such technology, where the injection of reference data in the system is transparent to the user. These interfaces are addressed by Internet Protocol (IP) stack and facilitate the process of sending and receiving data. Thus, for an application to send or receive data, it only needs to address the data packets with the IP address of the desired interface;

3. FIFO (First In First Out): due to the large data flow that must be supported by a LTE physical layer, the proposed framework provides a high-capacity data queue to avoid bottlenecks in both the acquisition and consumption of such data. The data queue also ensures that they can be consumed at a data rate different from that used during the generation. Virtual network interfaces allow their data to be redirected to other applications, such functionality is known as Bridge (or data bridge). Through a bridge, the data received or sent to the virtual network interface architecture is captured/received by a FIFO, from where they can be consumed by the simplified medium access control layer (MAC LITE); 
4. Simplified MAC (MAC LITE): it is the central mechanism of the proposed framework. It acts as a simplified access layer, performing all startup procedures and execution of stability tests, following the standards pointed by the Femto Forum API. Through a series of parameters passed to this layer via a data base (not detailed in Figure 2), it performs both the data injection and operation of the physical layers attached to it. The simplified access layer supports parallel operation of multiple physical layers, which enables testing carrier aggregation (CA) technology, a key LTE-Advanced feature. In this technology, each carrier is generated by a different physical layer, being the role of the MAC LITE to define of which data is sent to each one of the attached physical layers.

5. Physical Layer Abstraction: it was developed so that different communication interfaces between the MAC and PHY are supported. The communication interfaces adopted for formatting the messages and procedures is part of the Femto Forum API (FAPI) reference. However, FAPI does not define any protocol for sending the messages to the physical layer, and therefore, UDP sockets, shared memory, PCI, among others, can be used to that end. The proposed framework provides abstraction on specific messages transmission to the LTE physical layer, leaving the message packaging to send or receive data up to the developer;

6. Physical Layer: this is the LTE physical layer under test. It may be commercial or under development solutions (the way the framework behaves for each type of physical layer will be explained in more detail in the following sections). Such physical layer must be compatible with the communication protocol defined by the Femto Forum API reference [23]. As previously mentioned, the way data messages are transported is not part of the scope of this article, being directly related to the technology adopted at the physical layer.

The process of communication between the upper layers and the physical layer must meet critical requirements of timing and minimum throughput. Such requirements must be tested during the integration process, and can also be done through the proposed architecture. The following section will detail the main use cases of the proposed architecture.

\subsection{Key Uses for the Proposed Framework}

The principal users of the proposed framework can be divided into two main groups:

- Developers integrating physical layer with other layers;

- Developers who are creating physical layers based on re-configurable or related hardware.

The main difference between the two groups of users lies in the fact that for the first group it is supposed that the physical layer is fully functional, and 
in this case, the framework is only used to test the messaging protocol. Figure 3 shows a basic environment based on the proposed framework for such user groups. It should be noted that a tool for capturing and analyzing messages is needed (e.g., Ethereal tool). This tool is not part of the scope of the proposed architecture as it depends on the way messages are exchanged between layers. Through this tool, users can evaluate the behavior of the physical layer under test, leaving the developer to adapt the access layer according to such behavior. These tests are useful to check problems in both timing and order of received messages exchanged between the physical and access layers. It starts from the assumption that the physical layer uses the Femto Forum API protocol for communication.

The second group, in turn, may use the architecture to check the operation of each one of the physical data channels (as will be explained in the next section), as part of the throughput and scalability tests of the physical layer. For this group of users it is necessary to use an external device for checking data integrity, such equipment is known as spectrum analyzer. Figure 4 shows a basic environment for developers testing physical layers.

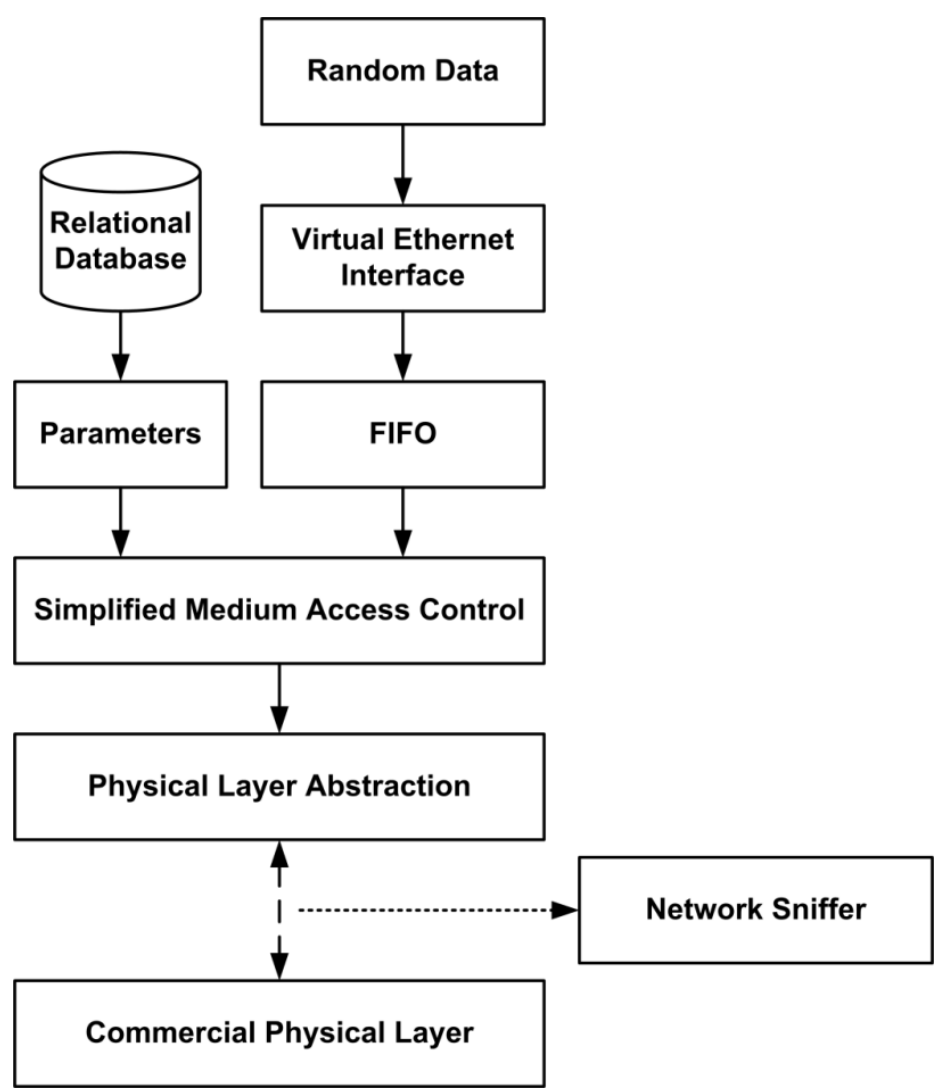

Fig. 3 Use case for the integration between the Access and Physical layers. 


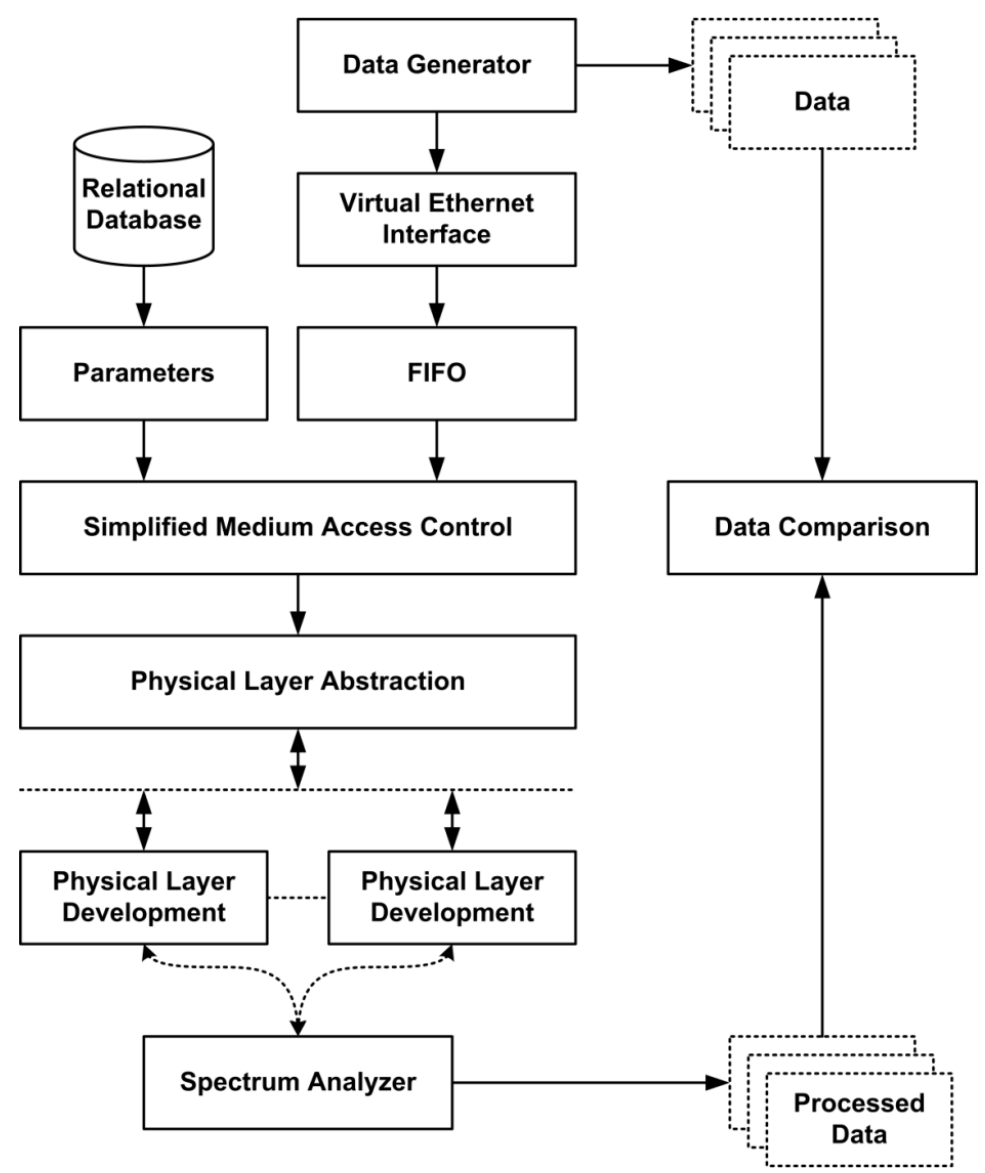

Fig. 4 Use case for Physical Layer Development.

\section{Fundamentals of the Proposed Framework}

As described in Figures 2, 3 and 4, one of the key components of proposed framework in this article is the Simplified MAC Layer. The LTE MAC layer is responsible for the following functionalities:

- Mapping between the logical and physical channels;

- Receiving and sending data from upper to physical layers;

- User data scheduling for serving the mobile terminals through the physical layer.

The Simplified MAC Layer performs the three functionalities above, but with no real integration with upper layers, since the mapping between logical and physical channels happens in an arbitrary manner, according to the user definition or type of test. In this case, the scheduling of mobile users is static and a list of user identifiers defines which users will be able to receive data from the FIFO. Data is sent from the FIFO to the users according to the 


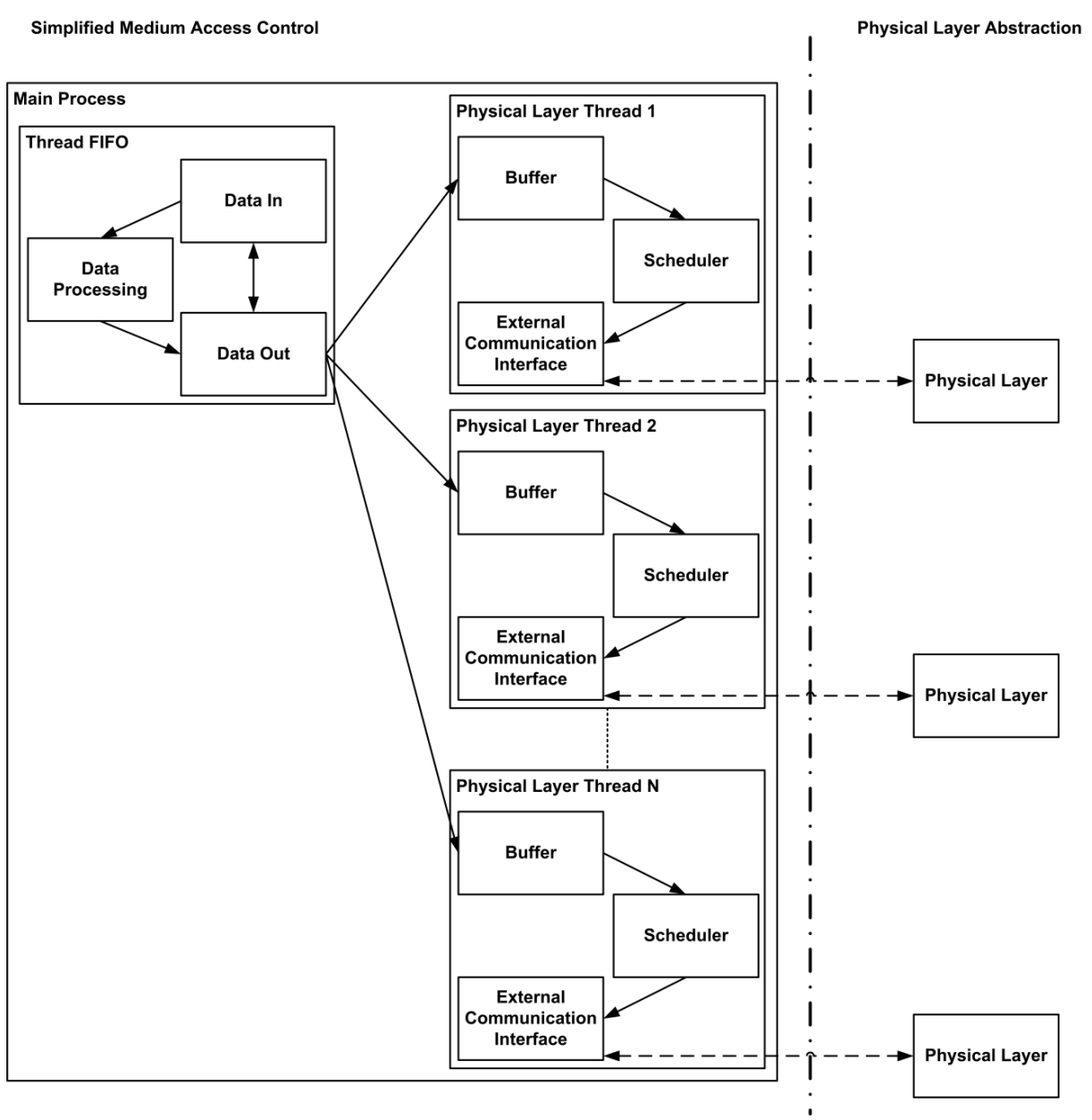

Fig. 5 Architecture proposed for the Simplified MAC.

feeding throughput from the FIFO, i.e., the maximum data rate supported by the mobile device. Such layer was designed to support the Carrier Aggregation technology, where the access layer needs to establish communication with various physical layers. In order to prevent performance and high latency communication problems, the proposed framework has been designed with support for multi-processing, i.e., multi-threading, where each CPU core carries out the operations of a physical layer, obtaining maximum performance of multi-processor computer architectures. Figure 5 shows the proposed architecture in more detail. Each physical layer is controlled by a separate execution thread, and the FIFO is controlled by a dedicated thread.

It is possible to see in Figure 5 two basic types of execution threads:

1. FIFO Thread: it is responsible for processing input and output data from the FIFO. Data reception is executed by the Data In module. The input 


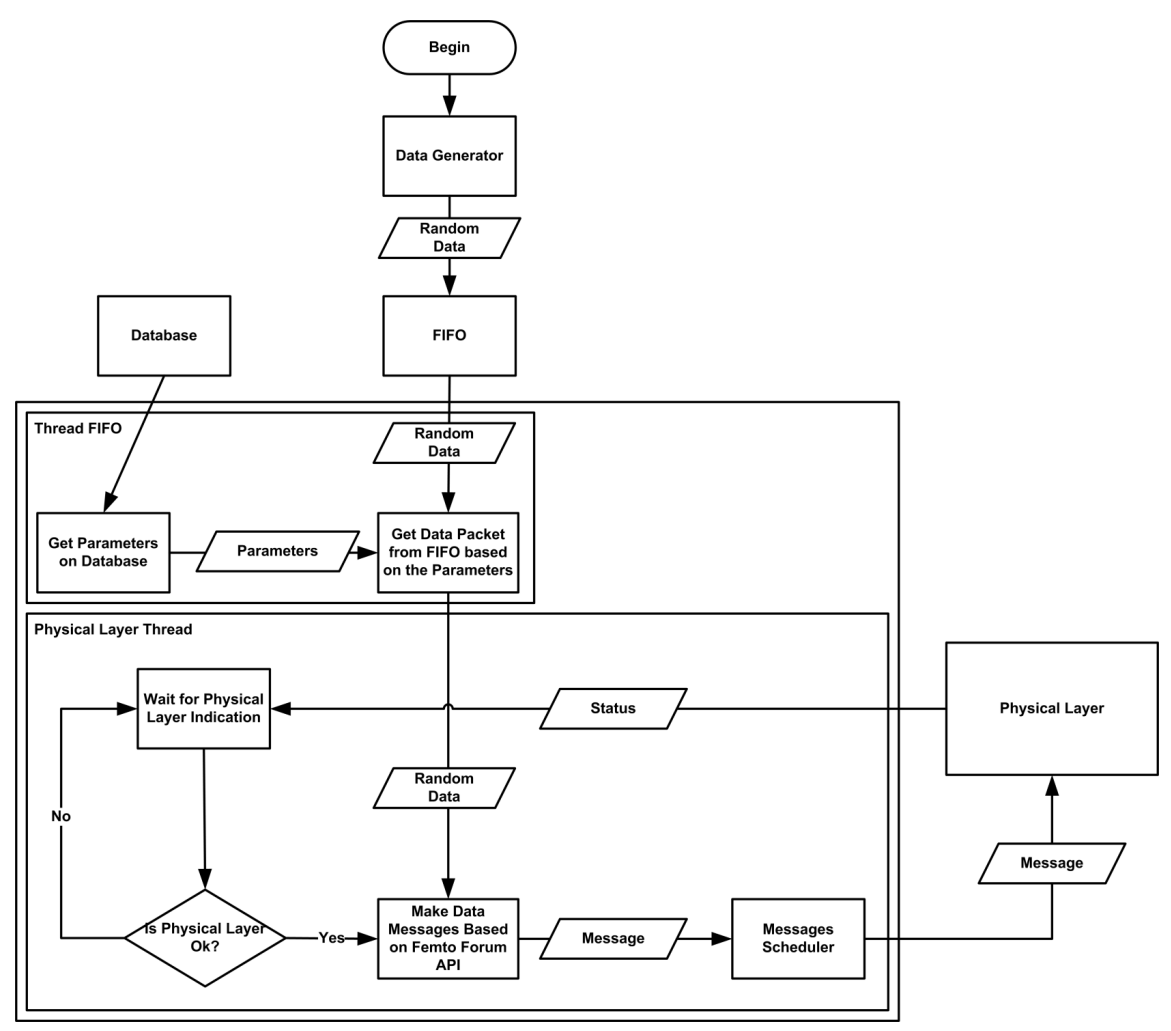

Fig. 6 Flowchart of the Simplified MAC Layer.

data comes from the virtual Ethernet interface, which was omitted in Figure 5 . The data is packaged by the Data Processing Module and divided into blocks with sizes that are integer multiples of the transmission capacity supported by the communication channel between the physical layer and the mobile terminals. Such data blocks are sent to a memory shared among threads named Data Output, which can be consumed by threads responsible for the operation of the physical layer. For simplification purposes, the data flow showed in Figure 5 only presents the data transmission in the direction of the physical layers, and they may also flow in the reverse direction, i.e., it is received by the physical layer through the mobile terminals;

2. Physical Layer Thread: it is responsible for interfacing with the physical layer. It reads the data stored in the FIFO output buffer thread and sends them to the physical layer. A thread of this kind is instantiated for each physical layer, due to the high data throughput required for operation of the LTE system. Another case of multiple threads is in the Carrier Aggregation functionality, where a single base station can simultaneously operate with multiple physical layers, each at a different carrier frequency. The buffer module constantly checks for data not consumed in the shared 
memory corresponding to the FIFO thread. If this is the case, the data is sent to the scheduler module, which waits the time of sending to the physical layer. The External Communication Interface module performs direct communication with the physical layer, exchanging data between the scheduler and the physical layer. It also performs all the communication process (synchronization procedure, sending and receiving data) through the Femto Forum API interface.

The joint operation of the threads described above provides all the basic operation of the proposed Simplified MAC Layer, whose operation is illustrated in more detail in the flowchart of Figure 6. The main steps are as follows:

1. The FIFO Thread accesses system configuration parameters that are stored in a relational database (database);

2. These parameters are used to define the limits of transmission and reception of the Physical Layer, such as rates and packets size;

3. With these parameters, the FIFO thread accesses the FIFO and formats the data packets (Random Data) with a size appropriate to the capacity of the Physical Layer;

4. Such packets are sent to the Physical Layer thread according to the rate set by the configuration parameters retrieved from the database;

5. On the Physical Layer thread side, the Physical Layer constantly sends indications of their status, informing when it is able to receive new data;

6. If so, the data packets (Random Data) provided by the FIFO are encapsulated into packets respecting the Femto Forum API format, and then are sent to the Message Scheduler;

7. The Message Scheduler forwards data messages in the Femto Forum API interface to the Physical Layer during test procedures;

8. The Physical Layer processes the message and returns a status message, restarting then the whole procedure;

9. The Physical Layer thread only sends new messages when the status indication provided by the Physical Layer indicates that it is able to receive new messages. Otherwise, the Physical Layer thread waits for this indication.

The Femto Forum API defines a set of error codes and status for many different situations. The status message returned by the Physical Layer is also used for indications of lack of synchronization, out of sequence messages and unexpected messages. All these indications are used so that the Simplified MAC Layer has more detailed information on the performance and stability of the Physical Layer during the testing procedures.

The main error indications are defined by the Femto Forum API communications protocol and include:

- Out of Sequence Message: Indicates that the Physical Layer received a message out of sequence, indicating that it is still processing the previous message. This fact indicates that the Physical Layer is being operated above its capacity, indicating potential performance limitations; 
- Unexpected Message: Indicates that the Physical layer is not yet ready to receive a particular message and it is likely out of synchronization. This may indicate the Physical Layer is unstable;

- Lack of synchronization: indicates that the message expected by the Physical Layer arrived outside the period in which it was expected. This may indicate problems of communication between the Physical Layer and the Access Layer, or even internal communication bottlenecks in the Physical Layer.

The messages mentioned above are stored in log files, which can be analyzed by the developers of the physical layer. With this, they can focus on major improvements or bug fixes that impact the performance and stability of the physical layer in question.

\section{Conclusion}

This paper proposes specific framework for testing Physical Layers during the development phase. It presents the implementation details of a Simplified MAC Layer, focused on conformance, stability, data integrity and performance testing. The article does not presents practical results since it consists of the description of a standard eNodeB protocol architecture and proposes a simplified test framework, aimed at meeting the possible demands originated during the development of eNodeB. This tool is particularly useful for developing specific features on Physical Layer and RF front end levels, such as in the case of LTE and LTE-Advanced networks for Band 31.

\section{Conflicts of Interest}

The authors declare that there are no conflicts of interest regarding the publication of this paper.

\section{Availability of data}

Data sharing is not applicable to this article as no new data were created or analyzed in this study.

\section{Funding}

This research received no specific grant from any funding agency in the public, commercial, or not-for-profit sectors. 


\section{References}

1. 3GPP TS 36.401, Evolved Universal Terrestrial Radio Access Network (E-UTRAN), September 2012.

2. 3GPP TR 36.913, Requirements for further advancements for Evolved Universal Terrestrial Radio Access (E-UTRA) (LTE-Advanced) - v\%.3.0, December 2009.

3. 3GPP TS 36.300, E-UTRA and E-UTRAN Overall Description - v8.0.0, September 2012 .

4. 3GPP TS 36.201, LTE Physical Layer - General Description - v1.0.0, August 2011.

5. 3GPP TS 36.323, Packet Data Convergence Protocol (PDCP) specification, December 2009.

6. 3GPP TS 36.322, Radio Link Control (RLC) protocol specification, March 2010.

7. 3GPP TS 36.321, Medium Access Control (MAC) protocol specification, March 2010.

8. 3GPP TS 36.331, Radio Resource Control (RRC); Protocol specification, March 2010.

9. 3GPP TS 36.213, Physical Layer Procedures, September 2012.

10. 3GPP TS 36.211, Physical Channels and Modulation - v1.0.0, September 2012.

11. 3GPP TS 36.214, Physical Layer - Measurements - v0.1.0, August 2011.

12. 3GPP TR 36.840, LTE 450MHz in Brazil Work Item Technical Report, September 2013.

13. Brazilian National Agency of Communications (ANATEL). Resolution No. 558, Regulations on channelization and use conditions of radio-frequencies in the 450-470 $\mathrm{MHz}$ band, December 2010.

14. Brazilian National Agency of Communications (ANATEL). The STEL System [Online]. Available: http://sistemas.anatel.gov.br/stel/

15. Brazilian Ministry of Communications, A national plan for broadband: Brazil in high speed, March 2009.

16. Sravanthi Kanchi, Shubhrika Sandilya, Deesha Bhosale, Adwait Pitkar, and Mayur Gondhalekar, Overview of LTE-A technology, IEEE Global High Tech Congress on Electronics (GHTCE), March 2014.

17. J. Zyren, Overview of the 3GPP Long Term Evolution Physical Layer, NXP White Paper, September 2007.

18. Stefania Sesia, Issam Toufik and Matthew Baker, LTE - The UMTS Long Term Evolution: From Theory to Practice, John Wiley \& Sons, 2011.

19. Ian F. Akyildiz, David M.Gutierrez-Estevez, and Elias Chavarria Reyes, The evolution to $4 G$ cellular systems: LTE-Advanced, Physical Communication, vol. 3, no. 4, pp. 217244, December 2010.

20. 3GPP TS 36.521-3, Evolved Universal Terrestrial Radio Access (E-UTRA); User Equipment (UE) conformance specification; Radio transmission and reception; Part 3: Radio Resource Management (RRM) conformance testing, September 2010.

21. Tim Godfrey, Long-Term Evolution Protocol: How the Standard Impacts Media Access Control, Freescale White Paper, October 2011.

22. 3GPP TS 36.212, Multiplexing and Channel Coding, September 2012.

23. Small Cell Forum, LTE eNB L1 API definition, March 2015. 